\title{
BUNDLES OF BANACH ALGEBRAS
}

\author{
J. W. KITCHEN \\ Department of Mathematics \\ Duke University \\ Durham, NC 27706 USA \\ and \\ D. A. ROBBINS \\ Department of Mathematics \\ Trinity College \\ Hartford, CT 06106 USA
}

(Recieved October 23, 1992 and in revised form April 20, 1993)

\begin{abstract}
We study bundles of Banach algebras $\pi: A \rightarrow \mathrm{X}$, where each fiber $\mathrm{A}_{\mathrm{X}}=$ $\pi^{-1}(\{x\})$ is a Banach algebra and $X$ is a compact Hausdorff space. In the case where all fibers are commutative, we investigate how the Gelfand representation of the section space algebra $\Gamma(\pi)$ relates to the Gelfand representation of the fibers. In the general case, we investigate how adjoining an identity to the bundle $\pi: \mathrm{A} \rightarrow \mathrm{X}$ relates to the standard adjunction of identities to the fibers.
\end{abstract}

KEYWORDS AND PHRASES: Banach bundle, bundle of Banach algebras, Gelfand representation, fiber space.

1991 AMS SUBJECT CLASSIFICATION CODES: $46 \mathrm{H} 15,46 \mathrm{H} 25,46 \mathrm{~J} 25$

\section{INTRODUCTION}

This paper continues the study of Banach bundles which has been pursued by the authors in a number of papers. This time, the focus is on bundles of Banach algebras. The reader is referred to [1] or [2] for general information about Banach bundles, and to [3] for the precise definition of bundles of Banach algebras which we use here.

We make the blanket assumption for this paper that the base space $\mathrm{X}$ for any Banach bundle $\pi: \mathrm{E} \rightarrow \mathrm{X}$ which we deal with is both compact and Hausdorff. As a result, all of our bundles are full; that is, for each $\mathrm{x} \in \mathrm{X}$, the fiber $\mathrm{E}_{\mathrm{X}}=\pi^{-1}(\{\mathrm{x}\})=\{\sigma(\mathrm{x}): \sigma \in \Gamma(\pi)\}$. (See [1, $\mathrm{p}$. 26])

The paper is divided into two sections. The first section deals with bundles of commutative Banach algebras. If $\pi: \mathrm{A} \rightarrow \mathrm{X}$ is a bundle of commutative Banach algebras, then the section space $\Gamma(\pi)$ is also a commutative Banach algebra under the pointwise operations. We study how the (usual) Gelfand representation of $\Gamma(\pi)$ relates to the Gelfand representations of the fibers $A_{x}=\pi^{-1}(\{x\})$. We show, in particular, that $\Delta(\Gamma(\pi))$, the maximal ideal space of $\Gamma(\pi)$, can be identified with the disjoint union of the maximal ideal spaces of the fibers. This generalizes a theorem of Rickart. 
The second section concerns the adjunction of identities. Suppose that $\pi: A \rightarrow X$ is a bundle of (possibly non-commutative) Banach algebras. We show that there is a bundle of Banach algebras $\pi^{\prime}: A^{\prime} \rightarrow X$ such that

1) for each $x \in X,\left(A^{\prime}\right)_{X}=\left(A_{x}\right)_{1}$, the algebra obtained by adjoining an identity to $A_{x}$; and

2) the section space $\Gamma\left(\pi^{\prime}\right)$ is an algebra with identity which contains (an isomorphic copy of) $\Gamma(\pi)$.

\section{BUNDLES OF COMMUTATIVE BANACH ALGEBRAS}

Consider a bundle $\pi: \mathrm{A} \rightarrow \mathrm{X}$ of commutative Banach algebras. We will show that there is a natural way of embedding the maximal ideal spaces of the fibers $A_{x}=\pi{ }^{-1}(\{x\})$ in the maximal ideal space of $\ell=\Gamma(\pi)$. Moreover, so embedded, the maximal ideal spaces $\Delta\left(A_{x}\right)$ provide a fibering of $\Delta(\mathcal{\Lambda})$. This result, Proposition 6, generalizes a theorem of Rickart [4].

To relate our result to that of Rickart, we consider first Banach algebras with identities and adopt the following definition.

DEFINITION 1: Let $\pi: \mathrm{A} \rightarrow \mathrm{X}$ be a bundle of commutative Banach algebras. We say that the bundle has an identity if

1) each fiber $A_{x}=\pi^{-1}(\{x\})$ has an identity $e_{x}$; and

2) the section e, defined by $e(x)=e_{x}$ for each $x \in X$, belongs to the section space $\Gamma(\pi)$ (and hence is the

identity for $\Gamma(\pi))$.

We now show that if $\pi: A \rightarrow X$ is a bundle with identity of commutative Banach algebras then the section space $\Gamma(\pi)$ fulfills the hypotheses of Theorem 3.2.2 of Rickart [4].

PROPOSITION 2: Let $\pi: \mathrm{A} \rightarrow \mathrm{X}$ be a bundle with identity of commutative Banach algebras. Then the algebra $\Lambda=\Gamma(\pi)$ is a subdirect sum sum algebra of the family of fibers $\left\{A_{X}: x \in X\right\}$ which satisfies conditions (i), (ii), and (iii) of Theorem 3.2.2 of [4], namely:

(i) $\ell$ contains the identity selection e defined above;

(ii) $\mathcal{\Lambda}$ is closed under (pointwise) multiplication by elements of $\mathrm{C}(\mathrm{X})$; and

(iii) for each $\sigma \in \mathcal{l}$, the map $\mathrm{x} \mapsto\|\sigma(\mathrm{x})\|$ is upper semicontinous on $\mathrm{X}$.

PROOF: Since $\Gamma(\pi)$ is a Banach algebra under the sup norm, it is a closed subalgebra of the full direct sum $\sum\left\{A_{X}: x \in X\right\}$ as defined by Rickart $[4, p$. 77]. Because the base space $X$ is compact and Hausdorff, the set of function values $\{\sigma(x): \sigma \in \Gamma(x)\}$ exhausts $A_{X}$ for each $x \in X$. Hence $\ell=\Gamma(\pi)$ is a subdirect algebra of the family $\left\{A_{x}: x \in X\right\}$. Condition (i) is simply our definition of the algebra bundle having an identity. Condition (ii) is part of what is meant by the assertion that $\Gamma(\pi)$ is a $\mathrm{C}(\mathrm{X})$-module, and condition (iii) holds for the sections of any bundle of Banach spaces. 000

The next result turns out to be a strengthened version of Rickart's theorem.

PROPOSITION 3: Let $\pi: \mathrm{A} \rightarrow \mathrm{X}$ be as above. Then $\Delta(\mathcal{\mu})$, the maximal ideal space of $\Lambda=\Gamma(x)$, can be identified with

$$
\Omega=\left\{(\mathrm{x}, \mathrm{h}): \mathrm{x} \in \mathrm{X}, \mathrm{h} \in \Delta\left(\mathrm{A}_{\mathbf{x}}\right)\right\},
$$

the disjoint union of the maximal ideal spaces of the fibers, in such a way that the Gelfand representation of $\ell$ is described by the equation

for all $(x, h) \in \Omega$ and all $\sigma \in \curlywedge$. Moreover,

$$
\widehat{\sigma}(\mathbf{x}, \mathbf{h})=[\sigma(\mathbf{x})]^{\hat{}}(\mathrm{h})
$$


(1) for each $x \in X$, the topology on $\Delta\left(A_{x}\right)$ is the same as the topology which $\Delta\left(A_{x}\right)$ inherits from $\Delta(\boldsymbol{l})$

$(=\Omega)$; and

(2) the coordinate projection $\mathrm{p}: \Omega \rightarrow \mathrm{X}$ is continuous and closed with respect to the topology which $\Omega$

inherits from $\Delta(\boldsymbol{\Omega})$.

Thus, $\Delta(\boldsymbol{\Lambda})=\Omega$ is a fibered space over $\mathrm{X}$ whose fibers are the maximal ideal spaces $\Delta\left(\mathrm{A}_{\mathbf{X}}\right)$.

PROOF: In [4, p. 129-130], Rickart shows that $\Delta(\mathcal{l})$ and $\Omega$ can be identified as point sets in such a way that the identity $\hat{\sigma}(\mathrm{x}, \mathrm{h})=[\sigma(\mathrm{x})]^{-}(\mathrm{h})$ holds.

We now prove assertions (1) and (2), which relate the topologies of the spaces $\Delta(\mathcal{\Lambda}), X$, and $\Delta\left(A_{x}\right)$.

To prove continuity of the map $\mathrm{p}: \Omega \rightarrow \mathrm{X}$, we consider a convergent net $\left\{\left(\mathrm{x}_{\alpha}, \mathrm{h}_{\alpha}\right)\right\}$ in the compact space $\Delta(\mathcal{l})=\Omega$, say $\lim \left(\mathrm{x}_{\alpha}, \mathrm{h}_{\alpha}\right)=(\mathrm{x}, \mathrm{h})$. We must prove that $\lim \mathrm{x}_{\alpha}=\mathrm{x}$, or equivalently that $\lim \phi\left(\mathrm{x}_{\alpha}\right)=\phi(\mathrm{x})$ for all $\phi \in \mathrm{C}(\mathrm{X})$ (since the topology of $\mathrm{X}$ is the same as the

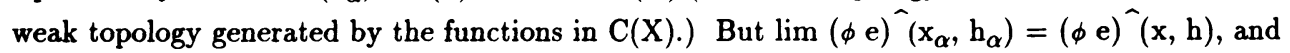
$(\phi \mathrm{e})^{\wedge}(\mathrm{x}, \mathrm{h})=[(\phi \mathrm{e})(\mathrm{x})]^{\hat{}}(\mathrm{h})=\left[\phi(\mathrm{x}) \mathrm{e}_{\mathrm{x}}\right]^{\hat{N}}(\mathrm{~h})=\phi(\mathrm{x}) \hat{\mathrm{e}}_{\mathbf{x}}(\mathrm{h})=\phi(\mathrm{x}) 1=\phi(\mathrm{x})$, and similarly $(\phi \mathrm{e})\left(\mathrm{x}_{\alpha}, \mathrm{h}_{\alpha}\right)=\phi\left(\mathrm{x}_{\alpha}\right)$. Thus, $\lim \phi\left(\mathrm{x}_{\alpha}\right)=\phi(\mathrm{x})$, as we wished to show. (A slightly more complicated proof appears later, when we no longer assume the existence of identity elements.)

To prove (1), it suffices to show that a net $\left\{h_{\alpha}\right\}$ in $\Delta\left(A_{X}\right)$ converges to a point $h$ iff the net $\left\{\left(x, h_{\alpha}\right)\right\}$ converges to $(x, h)$ in $\Delta(\mathcal{l})$. Assuming that $\lim h_{\alpha}=h$ in $\Delta\left(A_{x}\right)$, it follows that $\lim \hat{\sigma}\left(\mathrm{x}, \mathrm{h}_{\alpha}\right)=\lim [\sigma(\mathrm{x})]^{\wedge}\left(\mathrm{h}_{\alpha}\right)=[\sigma(\mathrm{x})]^{\wedge}(\mathrm{h})=\widehat{\sigma}(\mathrm{x}, \mathrm{h})$

for all $\sigma \in \ell$. If a is any element in $A_{x}$, we can choose $\sigma \in \ell$ such that $\sigma(x)=a$. Then, if $\lim \left(\mathrm{x}, \mathrm{h}_{\alpha}\right)=(\mathrm{x}, \mathrm{h})$ in $\Delta(\boldsymbol{\mu})$,

$\lim \hat{a}\left(h_{\alpha}\right)=\lim [\sigma(x)]^{\widehat{C}}\left(h_{\alpha}\right)=\lim \hat{\sigma}\left(x, h_{\alpha}\right)=\widehat{\sigma}(x, h)=[\sigma(x)]^{-}(h)=\hat{a}(h)$, which implies that $\lim h_{\alpha}=h$ in $\Delta\left(A_{x}\right)$. Because the algebra $\ell$ has an identity, the maximal ideal space $\Omega=\Delta(\Omega)$ is compact, and as a result the map p : $\Omega \rightarrow \mathrm{X}$ is not only continuous but closed. $\mathrm{uDO}$

Our next result shows that Proposition 3 is indeed a strengthened version of Rickart's theorem.

PROPOSITION 4: Let $X$ be a compact Hausdorff space, and let $\left\{A_{x}: x \in X\right\}$ be a family of commutative Banach algebras indexed by $\mathrm{X}$. Let $\boldsymbol{\Lambda}$ be a subdirect sum algebra of the family $\left\{A_{\mathbf{x}}: \mathbf{x} \in X\right\}$ which satisfies conditions (i), (ii), and (iii) of Proposition 2. Then the disjoint union

$$
A=\left\{(x, a): x \in X, a \in A_{x}\right\}
$$

can be uniquely topologized so that $\pi: \mathrm{A} \rightarrow \mathrm{X}$ is a bundle of Banach algebras having the elements of $\ell$ as sections (where $\pi$ is the obvious coordinate projection). Moreover, $l$ is the entire section space $\Gamma(\pi)$.

PROOF: The existence of the desired topology on $\ell$ follows from Proposition 1.3 in [2]. Because the norm on $\ell$ is a sup norm, it is easily shown that $l$ is $\mathrm{C}(\mathrm{X})$-locally convex as a module over $\mathrm{C}(\mathrm{X})$, and from this it follows that $\mathcal{\ell}=\Gamma(\pi)$. $\mathrm{\square} 0 \mathrm{D}$

COROLLARY 5: (Rickart's Theorem) Let $\left\{\mathrm{A}_{\mathrm{x}}: \mathrm{x} \in \mathrm{X}\right\}$ and $\mathcal{A}$ satisfy the conditions of Proposition 4. Then $\Delta(\mathcal{l})$ can be identified as a point set with the disjoint union

of the maximal ideal spaces of the algebras $A_{x}$.

$$
\Omega=\left\{(\mathrm{x}, \mathrm{h}): \mathrm{x} \in \mathrm{X}, \mathrm{h} \in \Delta\left(\mathrm{A}_{\mathrm{x}}\right)\right\}
$$


So, Proposition 3 is a strengthening of Rickart's result in this sense: not only can $\Delta(\mathcal{l})$ be identified as a point set with the disjoint union of the family $\left\{\Delta\left(A_{x}\right): x \in X\right\}$, but under this identification $\Delta\left(A_{X}\right)$ is a closed subset of $\Delta(\mathcal{A})$ for each $x$, and $\left\{\Delta\left(A_{X}\right): x \in X\right\}$ is an upper semicontinuous decomposition of $\Delta(\mathcal{l})$. We show next that much of this is true if we drop the assumption that the algebras in question have identities.

PROPOSITION 6: Let $\pi: \mathrm{A} \rightarrow \mathrm{X}$ be a bundle of commutative Banach algebras. Then $\Delta(\mathcal{l})$, the maximal ideal space of $\mathcal{\ell}=\Gamma(\pi)$, can be identified as a point set with the disjoint union

in such a way that

$$
\Omega=\left\{(\mathrm{x}, \mathrm{h}): \mathrm{x} \in \mathrm{X}, \mathrm{h} \in \Delta\left(\mathrm{A}_{\mathbf{x}}\right)\right\}
$$

$$
\widehat{\sigma}(\mathrm{x}, \mathrm{h})=[\sigma(\mathrm{x})]^{\widehat{ }}(\mathrm{h})
$$

holds for all $(\mathrm{x}, \mathrm{h}) \in \Omega$ and all $\sigma \in \Gamma(\pi)$. Moreover, under this identification, $\Delta\left(\mathrm{A}_{\mathrm{x}}\right)$ is a closed subset of $\Delta(\mathcal{\Lambda})$ for each $x \in X$.

PROOF: If $B$ is a commutative Banach algebra, we shall regard each point in $\Delta(B)$ as an algebra homomorphism from $B$ onto $C$.

Consider an element $(x, h) \in \Omega$. Since the evaluation map $\operatorname{ev}_{x}: \Gamma(\pi) \rightarrow A_{x}$, defined by $\mathbf{e v}_{\mathbf{x}}(\sigma)=\sigma(\mathbf{x})$ is a surjective algebra homomorphism, its composition with $\mathrm{h}: \mathrm{A}_{\mathbf{X}} \rightarrow \mathbf{C}$ gives us an algebra homomorphism $\phi_{x, h}=h^{\circ} e v_{x}$ of $\mathcal{\ell}=\Gamma(\pi)$ onto C. Thus, $\phi_{x, h}$ belongs to $\Delta(\mathcal{l})$, and for all $\sigma \in \ell$,

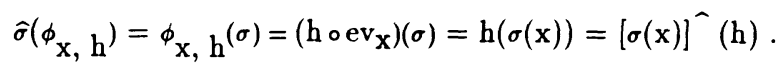

Let $\phi: \Omega \rightarrow \Delta(\mathcal{l})$ assign to each point $(\mathrm{x}, \mathrm{h})$ in $\Omega$ the multiplicative linear functional $\phi_{\mathrm{x}}, \mathrm{h}$ We must show that the map is bijective.

To show injectivity, consider two distinct points $\left(\mathrm{x}_{1}, \mathrm{~h}_{1}\right)$ and $\left(\mathrm{x}_{2}, \mathrm{~h}_{2}\right) \in \Omega$. We will show that $\phi_{\mathrm{x}_{1}, \mathrm{~h}_{1}} \neq \phi_{\mathrm{x}_{2}}, \mathrm{~h}_{2}$.

Case 1: $x_{1}=x_{2}$ and $h_{1} \neq h_{2}$. Because $h_{1} \neq h_{2}$, there is an $a \in A_{x}$ such that $h_{1}(a) \neq h_{2}(a)$. If we choose $\sigma \in \mathcal{l}$ such that $\sigma\left(\mathrm{x}_{1}\right)=\mathrm{a}$, then

$$
\phi_{\mathrm{x}_{1}, \mathrm{~h}_{1}}(\sigma)=\mathrm{h}_{1}\left(\sigma\left(\mathrm{x}_{1}\right)\right)=\mathrm{h}_{1}(\mathrm{a}) \neq \mathrm{h}_{2}(\mathrm{a})=\mathrm{h}_{2}\left(\sigma\left(\mathrm{x}_{2}\right)\right)=\phi_{\mathrm{x}_{2}, \mathrm{~h}_{2}}(\sigma) .
$$

Case 2: $\mathrm{x}_{1} \neq \mathrm{x}_{2}$. Choose $\mathrm{a} \in \mathrm{A}_{\mathrm{x}_{1}}$ such that $\mathrm{h}_{1}(\mathrm{a})=1$ and choose $\sigma \in \mathcal{1}$ such that $\sigma\left(\mathrm{x}_{1}\right)=$ a. Next choose $f \in C(X)$ such that $f\left(x_{1}\right)=1$ and $f\left(x_{2}\right)=0$. Then

and

$$
\phi_{\mathrm{x}_{1}, \mathrm{~h}_{1}}(\mathrm{f} \sigma)=\mathrm{h}_{1}\left((\mathrm{f} \sigma)\left(\mathrm{x}_{1}\right)\right)=\mathrm{h}_{1}\left(\mathrm{f}\left(\mathrm{x}_{1}\right) \sigma\left(\mathrm{x}_{1}\right)\right)=\mathrm{h}_{1}(\mathrm{a})=1
$$

Thus, $\phi_{\mathrm{x}_{1}, \mathrm{~h}_{1}} \neq \phi_{\mathrm{x}_{2}, \mathrm{~h}_{2}}$.

$$
\phi_{\mathrm{x}_{2}, \mathrm{~h}_{2}}(\mathrm{f} \sigma)=\mathrm{h}_{2}\left(\left(\mathrm{f}_{\sigma}\right)\left(\mathrm{x}_{2}\right)\right)=\mathrm{h}_{2}\left(\mathrm{f}\left(\mathrm{x}_{2}\right) \sigma\left(\mathrm{x}_{2}\right)\right)=\mathrm{h}_{2}(0)=0 .
$$

Having shown that the map $\phi: \Omega \rightarrow \Delta(\Omega)$ is injective, we show next that the map is surjective. Suppose, now, that $H \in \Delta(\mathcal{l})$. We choose $\sigma \in \mathcal{\ell}$ such that $H(\sigma)=1$ and we define a functional $\psi: \mathbf{C}(\mathbf{X}) \rightarrow \mathbf{C}$ by $\psi(\mathbf{f})=\mathrm{H}(\mathbf{f} \sigma)$. Then it is easily checked that $\psi$ is bounded and linear. Moreover, $\psi$ is multiplicative, since

$$
\psi(\mathrm{fg})=\mathrm{H}(\mathrm{fg} \sigma)=\mathrm{H}(\mathrm{fg} \sigma) \mathrm{H}(\sigma)=\mathrm{H}\left(\mathrm{fg} \sigma^{2}\right)=\mathrm{H}(\mathrm{f} \sigma) \mathrm{H}(\mathrm{g} \sigma)=\psi(\mathrm{f}) \psi(\mathrm{g})
$$

for all $\mathrm{f}, \mathrm{g} \in \mathrm{C}(\mathrm{X})$. Thus, $\psi \in \Delta(\mathrm{C}(\mathrm{X}))$ and, consequently, there is a unique $\mathrm{x} \in \mathrm{X}$ such that $\psi$ is evaluation at $x$. We have, then,

for all $f \in C(X)$.

$$
\mathrm{H}(\mathrm{f} \sigma)=\psi(\mathrm{f})=\mathrm{f}(\mathrm{x})
$$

We will now show that there exists an $h \in \Delta\left(A_{X}\right)$ such that $H=\phi_{X, h}$.

First, we review some facts about the natural embedding of $\left(A_{X}\right)^{*}$ into $\ell^{*}=\Gamma(\pi)^{*}$. The evaluation map $\mathrm{ev}_{\mathrm{X}}: \boldsymbol{l} \rightarrow \mathrm{A}_{\mathrm{X}}$ is a quotient map, and consequently the adjoint map 
$\left(e_{\mathbf{x}}\right)^{*}:\left(A_{\mathbf{x}}\right)^{*} \rightarrow \Lambda^{*}$ is an isometry. If $k \in\left(A_{x}\right)^{*}$ and $K=\left(e v_{x}\right)^{*}(k)$, then

$$
\mathrm{K}(\tau)=\left\{\left(\mathrm{ev}_{\mathbf{X}}^{*}(\mathrm{k})\right\}(\tau)=\mathrm{k}\left(\mathrm{ev}_{\mathbf{X}}(\tau)\right)=\mathrm{k}(\tau(\mathrm{x}))\right.
$$

for all $r \in \ell$. Thus, if $\mathrm{f} \in \mathrm{C}(\mathrm{X})$ and $\tau \in \mathcal{\ell}$,

$$
\mathrm{K}(\mathrm{f} \tau)=\mathrm{k}((\mathrm{f} \tau)(\mathrm{x}))=\mathrm{k}(\mathrm{f}(\mathrm{x}) \tau(\mathrm{x}))=\mathrm{f}(\mathrm{x}) \mathrm{k}(\tau(\mathrm{x}))=\mathrm{f}(\mathrm{x}) \mathrm{K}(\tau) .
$$

Conversely, one can show that if $K \in \boldsymbol{\Lambda}^{*}$ and if the equation

$$
K(f \tau)=f(x) K(\tau)
$$

holds for all $f \in C(X)$ and $\tau \in \mathcal{l}$, then there is a unique $k \in\left(A_{x}\right)^{*}$ such that $K=\left(e_{x}\right)^{*}(k)$. (See Proposition 2.1 of [2].)

Now, consider $\mathrm{H}$ again. If $\mathrm{f} \in \mathrm{C}(\mathrm{X})$ and $r \in \boldsymbol{l}$, then

$$
\mathrm{H}(\mathrm{f} \tau)=\mathrm{H}(\mathrm{f} \tau) \mathrm{H}(\sigma)=\mathrm{H}(\mathrm{f} \tau \sigma)=\mathrm{H}(\mathbf{f} \sigma) \mathrm{H}(\tau)=\mathrm{f}(\mathrm{x}) \mathrm{H}(\tau) .
$$

By the preceding paragraph, there is a unique $h \in\left(A_{\mathbf{x}}\right)^{*}$ such that $H=\left(e_{\mathbf{x}}\right)^{*}(h)$, and thus

$$
\mathrm{H}(\tau)=\mathrm{h}(\tau(\mathrm{x}))
$$

for all $\tau \in \mathcal{A}$. Since $\mathrm{H}$ is multiplicative, it immediately follows that $\mathrm{h}$ is multiplicative. Hence $\mathrm{h} \in \Delta\left(\mathrm{A}_{\mathbf{x}}\right)$ and

$$
\mathrm{H}(\tau)=\mathrm{h}(\tau(\mathbf{x}))=\phi_{\mathbf{x}, \mathbf{h}^{(\tau)}}
$$

We have now proved that $\Omega$ can be identified with $\Delta(\mathcal{\Lambda})$ in such a way that

$$
\hat{\sigma}(\mathbf{x}, \mathbf{h})=\hat{\sigma}\left(\phi_{\mathbf{x}, \mathbf{h}}\right)=\mathrm{h}(\sigma(\mathbf{x}))=[\boldsymbol{\sigma}(\mathbf{x})]^{-}(\mathbf{h})
$$

for all $(\mathrm{x}, \mathrm{h}) \in \Omega$ and $\sigma \in \mathcal{A}$. From this it follows that the topology on $\Delta\left(\mathrm{A}_{\mathrm{x}}\right)$ is the same as the topology which it inherits as a subspace of $\Delta(\Omega)$. (See the proof of condition 1) in Proposition 3.)

We show finally that the map $p: \Delta(\Lambda) \rightarrow X$ is continuous. Suppose that $\left\{\left(x_{\alpha}, h_{\alpha}\right)\right\}$ is a net in $\Omega=\Delta(\mathcal{l})$ which converges to a point $(x, h)$. Thus

$$
\lim \widehat{\sigma}\left(\mathrm{x}_{\alpha}, \mathrm{h}_{\alpha}\right)=\lim \mathrm{h}_{\alpha}\left(\sigma\left(\mathrm{x}_{\alpha}\right)\right)=\widehat{\sigma}(\mathrm{x}, \mathrm{h})=\mathrm{h}(\sigma(\mathrm{x}))
$$

for all $\sigma \in \mathcal{l}$. Choose $\mathrm{a} \in \mathrm{A}_{\mathrm{X}}$ such that $\mathrm{h}(\mathrm{a})=1$ and choose $\tau \in \ell$ such that $\tau(\mathrm{x})=\mathrm{a}$. Then

$$
(*) \lim \mathrm{h}_{\alpha}\left(\tau\left(\mathrm{x}_{\alpha}\right)\right)=\mathrm{h}(\tau(\mathrm{x}))=\mathrm{h}(\mathrm{a})=1 \text {. }
$$

Let $\phi \in C(X)$. Then

$$
\lim \mathrm{h}_{\alpha}\left((\phi \tau)\left(\mathrm{x}_{\alpha}\right)\right)=\mathrm{h}((\phi \tau)(\mathrm{x})) .
$$

But $\mathrm{h}((\phi \tau)(\mathrm{x}))=\mathrm{h}(\phi(\mathrm{x}) \tau(\mathrm{x}))=\phi(\mathrm{x}) \mathrm{h}(\tau(\mathrm{x}))=\phi(\mathrm{x})$, and similarly $\mathrm{h}_{\alpha}\left((\phi \tau)\left(\mathrm{x}_{\alpha}\right)\right)=\phi\left(\mathrm{x}_{\alpha}\right)$ $\mathrm{h}_{\alpha}\left(\tau\left(\mathrm{x}_{\alpha}\right)\right)$. Thus

$$
(* *) \lim \phi\left(\mathrm{x}_{\alpha}\right) \mathrm{h}_{\alpha}\left(\tau\left(\mathrm{x}_{\alpha}\right)\right)=\phi(\mathrm{x}) .
$$

From $(*)$ and $(* *)$ it follows that

\section{Hence $\lim x_{\alpha}=x$ in X. 000}

$$
\lim \phi\left(\mathrm{x}_{\alpha}\right)=\lim \frac{\phi\left(\mathrm{x}_{\alpha}\right) \mathrm{h}_{\alpha}\left(\tau\left(\mathrm{x}_{\alpha}\right)\right)}{\mathrm{h}_{\alpha}\left(\tau\left(\mathrm{x}_{\alpha}\right)\right)}=\frac{\phi(\mathrm{x})}{1}=\phi(\mathrm{x}) .
$$

The reader will note that there is a result analogous to Proposition 6 to be found in the theory of bundles of $\mathrm{C}^{*}$-algebras; see, for example [5, p. 582]. Namely, if $\pi: \mathrm{A} \rightarrow \mathrm{X}$ is a bundle of $\mathrm{C}^{*}$-algebras (with the total space A having continuous norm), then for every irreducible *representation $\mathrm{T}$ of the $\mathrm{C}^{*}$-algebra $\Gamma(\pi)$, there exists $\mathrm{x} \in \mathrm{X}$ and an irreducible ${ }^{*}$-representation $\mathrm{S}$ of $A_{X}$ such that $T=S^{\circ} e_{x}$. The proof of Proposition 6 (which has an evident corollary in common with the $\mathrm{C}^{*}$-algebra result) requires neither the ${ }^{*}$-machinery nor the existence of approximate identities which are used in [5], but, of course, does not deal with non-commutative algebras .

We consider next two examples. In the first, we show that the map $p: \Delta(\mathcal{\Lambda}) \rightarrow \mathrm{X}$ need not be closed, even if all the fibers $A_{X}$ have identities. In the second, we show that the map $p: \Delta(\Lambda)$ $\rightarrow \mathrm{X}$ need not be open, even if $\pi: \mathrm{A} \rightarrow \mathrm{X}$ is a bundle with identity. 
EXAMPLE 7: Let $X=[0,1]$ and for each $x \in X$ let $A_{X}$ be the one-dimensional Banach algebra $C$ normed by absolute value. Then the full direct sum of the family $\left\{A_{x}: x \in X\right\}$ is the space $\ell^{\infty}(\mathrm{X})$ of all bounded complex-valued functions on $\mathrm{X}$. We let $\ell$ be the subalgebra consisting of all functions $f:[0,1] \rightarrow C$ such that $f$ is continuous on $[0,1)$ and $\lim _{x \rightarrow 1}-f(x)=0$ ( and $\mathrm{f}(1)$ is arbitrary). Then it is easily verified that $\ell$ is a subdirect sum algebra of $\ell^{\infty}(\mathrm{X})$ which satisfies conditions (ii) and (iii) of Proposition 2, but not condition (i). (Note that if $f \in \ell$, then the function $x \mapsto\|f(x)\|=|f(x)|$ is upper semicontinuous on $[0,1]$. On the other hand, the identity selection $\mathrm{e}(\mathrm{x}) \equiv 1$ does not belong to 1 .)

It is easy to show that $\Delta(\Lambda)$ consists of the evaluation functionals $e_{x}$ for $x \in[0,1]$. Thus, as a point set, $\Delta(\Lambda)$ can be identified with $[0,1]$. The topologies, however, do not match. In [0, 1] the sequence $\{(n-1) / n\}$ converges to 1 , whereas in $\Delta(\mathcal{l})$ the corresponding sequence $\left\{\mathrm{ev}_{(\mathrm{n}-1) / \mathrm{n}}\right\}$ has no limit. (In $\boldsymbol{\Lambda}^{*}$ the sequence converges weak-* to the zero functional.) In $\Delta(\boldsymbol{\Lambda})$ the point $\mathrm{ev}_{1}$ is isolated. The natural surjection $\mathrm{p}: \Delta(\mathcal{\Lambda}) \rightarrow \mathrm{X}$ maps $\mathrm{ev}_{\mathrm{X}}$ onto $\mathrm{x}$ for each $\mathrm{x} \in[0,1]$ . However, $p$ is not closed: the set $\left\{\operatorname{ev}_{x}: 0 \leq x<1\right\}$ is the complement of the open set $\left\{e_{1}\right\}$ and hence is closed. Its image under $p$ is $[0,1)$, and the latter is not closed in $[0,1]$.

EXAMPLE 8: Let $q: Y \rightarrow X$ be a continuous surjective map, where $X$ and $Y$ are compact Hausdorff. For each $x \in X$, set $Y_{x}=q^{-1}(\{x\})$ and $A_{x}=C\left(Y_{x}\right)$. Given $f \in C(Y)$ and $x \in X$, we define $f^{*}(x)$ to be the restriction of $f$ to $Y_{x}$. In this way, $l=C(Y)$ can be viewed as a subdirect sum of the family $\left\{A_{x}: x \in X\right\}=\left\{C\left(Y_{X}\right): x \in X\right\}$. (See [6] or [7].) Moreover, $\mathcal{A}$ satisfies conditions (i), (ii), and (iii) of Proposition 2. The maximal ideal space of $\curlywedge=C(Y)$ can be identified with $Y$, which in turn can be identified with

$$
\Omega=\left\{(\mathrm{x}, \mathrm{y}): \mathrm{x} \in \mathrm{X}, \mathrm{y} \in \mathrm{Y}_{\mathrm{x}}\right\} \text {. }
$$

Now, for each $(x, y) \in \Omega$,

$$
\mathrm{p}((\mathrm{x}, \mathrm{y}))=\mathrm{x}=\mathrm{q}(\mathrm{y}) .
$$

That is, under the identification of $\Delta(\mathcal{l})$ with $\mathrm{Y}$, the map $\mathrm{p}: \Delta(\mathcal{l}) \rightarrow \mathrm{X}$ is the same as the given map $q: Y \rightarrow X$. Since q may not be open, the map $p: \Delta(\mathcal{Y}) \rightarrow \mathrm{X}$ need not be open. (For example, let $Y=[-1,2], X=[0,4]$, and $q(x)=x^{2}$. Then the set $[-1,1 / 2)$ is open in $Y$, but its image under $q$ is $[0,1]$, which is not open in $[0,4]$.)

We close this section with some simple consequences of our main result.

PROPOSITION 9: Let $\pi: \mathrm{A} \rightarrow \mathrm{X}$ be a bundle of commutative Banach algebras.

(1) If $A_{X}$ is semi-simple for each $x \in X$, then $\Gamma(x)$ is semi-simple.

(2) Under our identification of $\Delta(\Gamma(\pi))$ with $\Omega=\left\{(\mathrm{x}, \mathrm{h}): \mathrm{x} \in \mathrm{X}, \mathrm{h} \in \Delta\left(\mathrm{A}_{\mathrm{x}}\right)\right\}$, the Silov boundary of $\Gamma(\pi)$ is the disjoint union of the Silov boundaries of the fibers $A_{x}$.

(3) If $\pi: \mathrm{A} \rightarrow \mathrm{X}$ is a bundle with identity, then for $\sigma \in \Gamma(\pi)$, the spectrum of $\sigma$ is the union of the spectra of the section values $\sigma(x)$; if $\pi: A \rightarrow X$ is a bundle without identity, then for $\sigma \in \Gamma(\pi)$, the spectrum of $\sigma$ is the union of the spectra of the section values $\sigma(x)$ and $\{0\}$. PROOF: All three assertions follow easily from the identity $\hat{\sigma}(\mathrm{x}, \mathrm{h})=[\sigma(\mathrm{x})]^{-}(\mathrm{h})$. We omit the details. 000

\section{THE ADJUNCTION OF IDENTITIES TO BUNDLES OF BANACH ALGEBRAS}

If $B$ is a (complex) Banach algebra, then there is a standard way of embedding $B$ into a Banach algebra with identity. We let $B_{1}$ be the vector space $B \times C$ with norm and multiplicaton defined by

$$
|(a, \lambda)|=\|\mathrm{a}\|+|\lambda| \quad \text { and } \quad(a, \lambda)(b, \mu)=(a b+\lambda b+\mu a, \lambda \mu) .
$$


Then $B_{1}$ is a Banach algebra with identity (the pair $(0,1)$ ) which contains a subalgebra, namely $\mathrm{B} \times\{0\}$, which is isometrically isomorphic to $\mathrm{B}$.

PROPOSITION 10: Let $\pi: \mathrm{A} \rightarrow \mathrm{X}$ be a bundle of Banach algebras. Then there exists a bundle $\pi^{\prime}: A^{\prime} \rightarrow X$ of Banach algebras such that

(1) for each $x \in X,\left(A^{\prime}\right)_{X}=\left(A_{X}\right)_{1}$;

(2) for all $\sigma \in \Gamma(x)$ and $f \in C(X)$, the selection $(\sigma, f) \sim: X \rightarrow A^{\prime}$ defined by $(\sigma, f) \sim(x)=$ $(\sigma(x), f(x))$

belongs to the section space $\Gamma\left(x^{\prime}\right) ;$ and

(3) $\Gamma\left(x^{\prime}\right)$ is a Banach algebra with identity which contains subalgebras isometrically isomorphic to $\Gamma(\pi)$,

$\{\Gamma(\pi)\}_{1}$, and $C(X)$.

PROOF: We let $M=\Gamma(\pi) \times C(X)$. Then it is easily verified that $M$ becomes a Banach algebra if the norm and multiplication are defined as follows:

(We have, for instance,

$$
\|(\sigma, \mathrm{f})\|=\|\sigma\|+\|\mathrm{f}\|, \quad \text { and } \quad(\sigma, \mathrm{f})(\tau, \mathrm{g})=(\sigma \tau+\mathrm{f} \tau+\mathrm{g} \sigma, \mathrm{fg}) .
$$

$$
\begin{aligned}
\|(\sigma, \mathrm{f})(\tau, \mathrm{g})\|= & \|\sigma \tau+\mathrm{f} \tau+\mathrm{g} \sigma\|+\|\mathrm{fg}\| \\
& \leq\|\sigma \tau\|+\|\mathrm{f} \tau\|+\|\mathrm{g} \sigma\|+\|\mathrm{fg}\| \\
& \leq\|\sigma\|\|\tau\|+\|\mathrm{f}\|\|\tau\|+\|\mathrm{g}\|\|\sigma\|+\|\mathrm{f}\|\|\mathrm{g}\| \\
& =(\|\sigma\|+\|\mathrm{f}\|)(\|\tau\|+\|\mathrm{g}\|) \\
& =\|(\sigma, \mathrm{f})\|\|(\tau, \mathrm{g})\| \cdot)
\end{aligned}
$$

Moreover, if we define $f(\tau, g)=(f \tau, f g)$ for all $f \in C(X)$ and $(\tau, g) \in M$, then $M$ becomes a $C(X)$ module. We let $\mu: \mathrm{B} \rightarrow \mathrm{X}$ be the canonical bundle of $\mathrm{M}$ as a $\mathrm{C}(\mathrm{X})$-module. We will show that $\mu: B \rightarrow X$ is an isomorphic copy of the bundle $\pi^{\prime}: A^{\prime} \rightarrow X$ which we seek.

Recall that for each $x \in X$, the fiber $B_{x}=\mu^{-1}(\{x\})$ is the quotient space $\frac{M}{I_{X} M}$, where $I_{x} M=\left\{f(\sigma, g): f \in I_{X},(\sigma, g) \in M\right\}$ and $I_{X}$ is the maximal ideal $\left\{f \in C(X): f(x) \stackrel{x^{\prime}}{=} 0\right\}$ in $C(X)$. (The set $I_{\mathbf{X}} M$ can also be characterized as the smallest closed subspace $J_{X}$ of $M$ which contains all products of the form $f(\sigma, g)$, where $f \in I_{X}$ and $(\sigma, g) \in M$. For $J_{X}$ is clearly an essential $I_{x}$ module, and the Banach algebra $I_{\mathbf{X}}$ has an approximate identity, so the Cohen factorization theorem implies that $J_{X}=I_{X} M$.) Moreover, the Gelfand sectional representation $\wedge: M \rightarrow \Gamma(\mu)$ is described by

$$
(\sigma, \hat{f})(x)=\Pi_{x}((\sigma, f))
$$

for all $(\sigma, f) \in M$, where $\Pi_{x}: M \rightarrow \frac{M}{I_{x} M}$ is the natural surjection. In this case, the set $I_{x} M$ is a closed two-sided ideal in $M$, so that $B_{x}=\frac{M}{I_{x} M}$ is a Banach algebra and $\Pi_{x}$ is an algebra homomorphism.

We will now show that for each $\mathrm{x} \in \mathrm{X}$ there is a unique isometric isomorphism $\phi_{\mathbf{X}}: \mathrm{B}_{\mathbf{X}} \rightarrow$ $\left(A_{x}\right)_{1}$ such that

$$
\phi_{\mathbf{X}}((\sigma, \hat{f}) \hat{(x)})=(\sigma(x), f(x))
$$

for all $(\sigma, f) \in M$. To that end, consider the map $\operatorname{ev}_{x}: M \rightarrow\left(A_{x}\right)_{1}$ defined by $\operatorname{ev}_{\mathbf{x}}((\sigma, \mathrm{f}))=(\sigma(\mathrm{x}), \mathrm{f}(\mathrm{x}))$. This map is evidently a norm-decreasing algebra homomorphism whose kernel includes $I_{X} M$. On the other hand, if $(\sigma, f) \in \operatorname{ker}\left(e_{X}\right)$, then $\sigma(x)=0$, so $\sigma \in I_{X} \Gamma(x)$; we also have $f(x)=0$, so $f \in I_{X}=I_{X} C(X)$. Hence $f$ and $\sigma$ have factorizations $f=h k$ and $\sigma=g r$, where $\mathrm{g}, \mathrm{h} \in \mathrm{I}_{\mathbf{X}}, \tau \in \Gamma(\pi)$, and $\mathrm{k} \in \mathrm{C}(\mathrm{X})$. It follows that $(\sigma, \mathrm{f})=\mathrm{g}(\tau, 0)+\mathrm{h}(0, \mathrm{k}) \in \mathrm{I}_{\mathbf{X}} \mathrm{M}$, since it is the sum of two elements of the subspace $I_{X} M$. Hence $k e r\left(e v_{X}\right)=I_{X} M$. 
Because ker $\left(\mathrm{ev}_{\mathbf{X}}\right)=\mathrm{I}_{\mathbf{X}} \mathrm{M}$, there is a unique injective algebra homomorphism $\phi_{\mathbf{X}}: \mathrm{B}_{\mathbf{X}} \rightarrow$ $\left(A_{x}\right)_{1}$

which makes the diagram

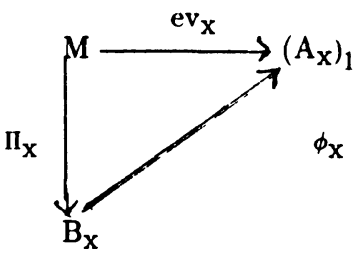

commute. Thus,

$$
\phi_{\mathbf{X}}((\sigma, \hat{f}) \hat{(x)})=\left(\phi_{\mathbf{X}} \circ \Pi_{\mathbf{X}}\right)(\sigma, f)=\operatorname{ev}_{\mathbf{X}}((\sigma, f))=(\sigma(\mathbf{x}), \mathfrak{f}(\mathbf{x}))
$$

for all $(\sigma, f) \in M$. To show that $\phi_{X}$ is an isometric isomorphism, it suffices to show that $e_{\mathbf{X}}: M$ $\rightarrow\left(A_{x}\right)_{1}$ is a quotient map. If $(z, \lambda)$ is any element in $\left(A_{x}\right)_{1}$, we can choose $\sigma \in \Gamma(\pi)$ and $f \in C(X)$ such that $\sigma(\mathrm{x})=\mathrm{z}, \mathrm{f}(\mathrm{x})=\lambda,\|\sigma\|=\|\sigma(\mathrm{x})\|=\|\mathrm{x}\|$, and $\|\mathrm{f}\|=|\mathrm{f}(\mathrm{x})|=|\lambda|$. (We could, for instance, let $f$ have the constant

value $\lambda$.) Then

$$
\operatorname{ev}_{\mathbf{x}}((\sigma, \mathrm{f}))=(\sigma(\mathrm{x}), \mathrm{f}(\mathrm{x}))=(\mathrm{z}, \lambda)
$$

and

$$
\|(\sigma, f)\|=\|\sigma\|+\|f\|=\|z\|+|\lambda|=\|(z, \lambda)\| .
$$

This proves that $\mathrm{ev}_{\mathbf{X}}: M \rightarrow\left(\mathrm{A}_{\mathbf{X}}\right)_{1}$ is a quotient map.

We now let $A^{\prime}$ be the disjoint union

$$
A^{\prime}=\left\{(x, z): x \in X, z \in\left(A_{x}\right)_{1}\right\}
$$

and let $\pi^{\prime}: \mathrm{A}^{\prime} \rightarrow \mathrm{X}$ be the coordinate projection. Then our family of isometric isomorphisms $\phi_{\mathbf{X}}: \mathrm{B}_{\mathbf{X}} \rightarrow\left(\mathrm{A}_{\mathbf{X}}\right)_{1}$ gives us a bijective map $\phi: \mathrm{B} \rightarrow \mathrm{A}^{\prime}$ which makes the diagram

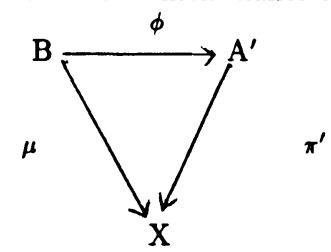

commute. (In other words, $\phi$ restricted to the fiber $B_{\mathbf{X}}$ is just $\phi_{\mathbf{X}}$.) We use the map $\phi$ to transfer the topology on the fiber space $B$ to $A^{\prime}$. Then $\pi^{\prime}: A^{\prime} \rightarrow X$ becomes a bundle of Banach algebras which is bundle isomorphic to $\mu: \mathrm{B} \rightarrow \mathrm{X}$. If $(\sigma, \mathrm{f}) \in \mathrm{M}$, then the selection $(\sigma, \mathrm{f}) \sim \mathrm{X} \rightarrow$ $\mathrm{A}^{\prime}$ defined by $(\sigma, \mathrm{f}) \sim(\mathrm{x})=(\sigma(\mathrm{x}), \mathrm{f}(\mathbf{x}))$ is just $\phi \circ(\sigma, \mathrm{f})^{\wedge}$ and hence is in the section space $\Gamma\left(\boldsymbol{\pi}^{\prime}\right)$.

The maps

$$
\begin{aligned}
& \alpha: \Gamma(\pi) \rightarrow \Gamma\left(\pi^{\prime}\right), \alpha(\sigma)=(\sigma, 0) ; \\
& \beta:\{\Gamma(\pi)\}_{1} \rightarrow \Gamma\left(\pi^{\prime}\right), \beta((\sigma, \lambda))=(\sigma, \lambda) \sim ; \text { and }
\end{aligned}
$$




$$
\gamma: \mathrm{C}(\mathrm{X}) \rightarrow \Gamma\left(\pi^{\prime}\right), \gamma(\mathrm{f})=(0, \mathrm{f}) \sim
$$

are clearly isometric algebra homomorphisms. 000

We conclude with an unrelated result.

If $\pi: \mathrm{A} \rightarrow \mathrm{X}$ is a bundle of Banach spaces, and if $\sigma \in \Gamma(\pi)$, then the numerical function $f(x)=\|\sigma(x)\|$ is upper semicontinuous on $X$. If $\pi: A \rightarrow X$ is a bundle of Banach algebras we will show that the same is true of the function $g(x)=\|\sigma(x)\|_{s p}$, where $\|$ a $\|_{\text {sp }}$ denotes the spectral radius of $\mathbf{a}$.

PROPOSITION 11: Let $\pi: \mathrm{A} \rightarrow \mathrm{X}$ be a bundle of Banach algebras, and let $\sigma \in \Gamma(\pi)$. Then the function $g(x)=\|\sigma(x)\| s p$ is upper semicontinuous on $X$. Moreover, if the algebras $\left\{A_{X}\right.$ : $\mathrm{x} \in \mathrm{X}\}$ are all commutative (so that $\Gamma(x)$ is commutative), then $\|\sigma\| \operatorname{sp}=\sup \{\|\sigma(\mathrm{x})\| \mathrm{sp}: \mathrm{x} \in \mathrm{X}\}$.

PROOF: Let $\mathrm{x}_{0} \in \mathrm{X}$, and let $\epsilon>0$ be given. Since

$$
\left\|\sigma\left(\mathrm{x}_{0}\right)\right\| \mathrm{sp}=\inf \left\|\left\{\sigma\left(\mathrm{x}_{0}\right)\right\}^{\mathrm{n}}\right\|^{1 / \mathrm{n}}=\lim \left\|\left\{\sigma\left(\mathrm{x}_{0}\right)\right\}^{\mathrm{n}}\right\|^{1 / \mathrm{n}}
$$

we can choose a positive integer $\mathrm{n}$ such that $\left\|\left\{\sigma\left(\mathrm{x}_{0}\right)\right\}^{\mathrm{n}}\right\|^{1 / \mathrm{n}}<\left\|\sigma\left(\mathrm{x}_{0}\right)\right\| \mathrm{sp}+\epsilon / 2$. Now, $\left\|\{\sigma(\mathbf{x})\}^{\mathrm{n}}\right\|=\left\|\sigma^{\mathrm{n}}(\mathrm{x})\right\|$ is an upper semicontinuous function of $\mathrm{x}$, and hence the same is true of $\left\|\{\sigma(x)\}^{n}\right\|^{1 / n}$. Thus, there is a neighborhood $V$ of $x_{0}$ such that

$$
\left\|\{\sigma(\mathrm{x})\}^{\mathrm{n}}\right\|^{1 / \mathrm{n}}<\left\|\left\{\sigma\left(\mathrm{x}_{0}\right)\right\}^{\mathrm{n}}\right\|^{1 / \mathrm{n}}+\epsilon / 2
$$

for all $x \in V$. Consequently,

$$
\|\sigma(\mathrm{x})\| \mathrm{sp} \leq\left\|\{\sigma(\mathrm{x})\}^{\mathrm{n}}\right\|^{1 / \mathrm{n}}<\left\|\sigma\left(\mathrm{x}_{0}\right)\right\| \mathrm{sp}+\epsilon
$$

for all $x \in V$, thus proving that the function $g(x)=\|\sigma(x)\|_{\text {sp }}$ is upper semicontinuous at $x_{0}$.

The assertion in the case when all algebras $A_{x}$ are commutative follows easily from 1 ) Proposition 6;

2) the fact that, if $B$ is any Banach algebra, then for $b \in B$, we have

$\|b\|_{\text {sp }}=\max \{|\lambda|: \lambda \in \operatorname{Sp}(b)\}$ (where $\operatorname{Sp}(b)$ denotes the spectrum of $b$ ) (see e.g. $[8$, p. 23]); and 3) the fact that, if $B$ is a commutative Banach algebra, and if $b \in B$, then the range of its Gelfand transform $\hat{b}$ is either $S p(b)$ or $S p(b) \backslash\{0\}$.

ACKNOWLEDGMENT: The authors wish to thank the referee for his careful reading and suggestions.

\section{REFERENCES}

1. GIERZ, G., Bundles of topological vector spaces and their duality, Lecture Notes in Math. 955, Berlin: Springer-Verlag, 1982.

2. KITHCEN, J.W. \& ROBBINS, D.A., Gelfand representation of Banach modules, Dissertationes Math. (Rozprawy Mat.) 203 (1982) (1983).

3. $---\frac{-}{135-156 .}$, Sectional representation of Banach modules, Pacific J. Math. 109 (1983), 
4. RICKART, C.E., General theory of Banach algebras, New York: Van Nostrand-Reinhold, 1960.

5. FELL, J.M.G. \& DORAN, R.S., Representations of *-algebras, locally compact groups, and Banach *-algebraic bundles, Boston: Academic Press, 1988.

6. KITHCEN, J.W. \& ROBBINS, D.A., Internal functionals and bundle duals, International J. Math. and Math. Sci. 7 (1984), 689-695.

7. SEDA, A., Banach bundles of continuous functions and an integral representation theorem, Trans. Amer. Math. Soc. 270 (1982), 327-332.

8. BONSALL, A. \& DUNCAN, J., Complete normed algebras, Berlin: Springer-Verlag, 1973. 


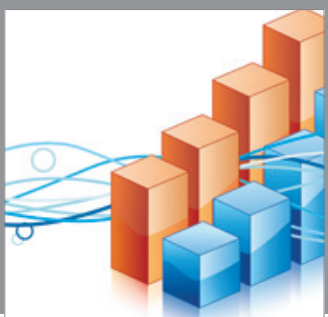

Advances in

Operations Research

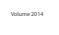

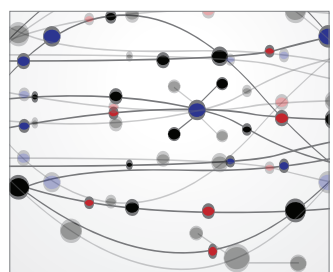

\section{The Scientific} World Journal
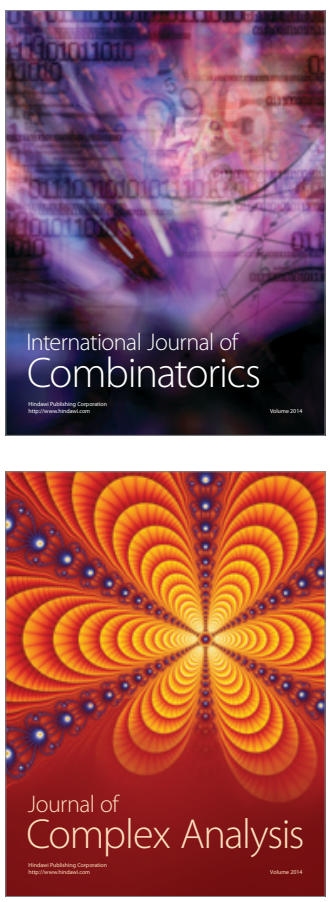

International Journal of

Mathematics and

Mathematical

Sciences
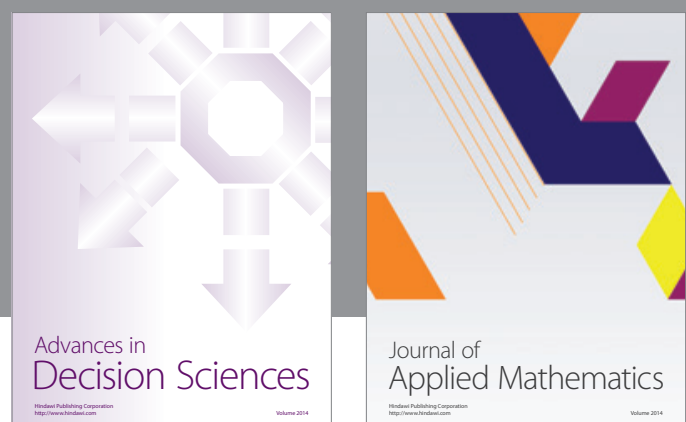

Journal of

Applied Mathematics
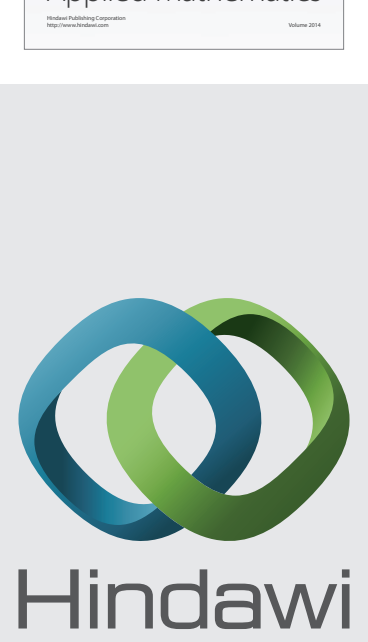

Submit your manuscripts at http://www.hindawi.com
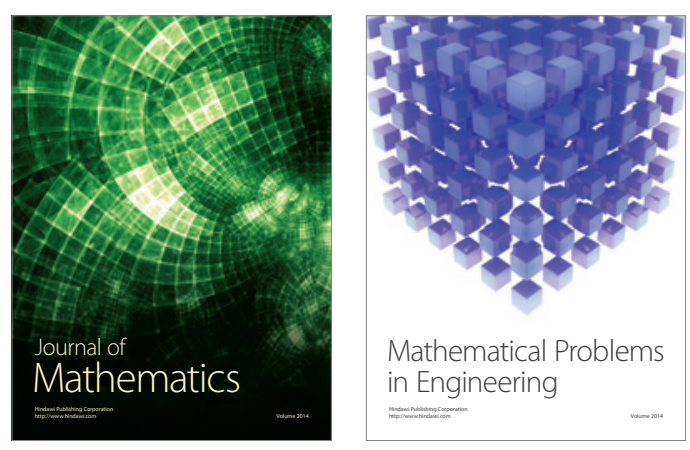

Mathematical Problems in Engineering
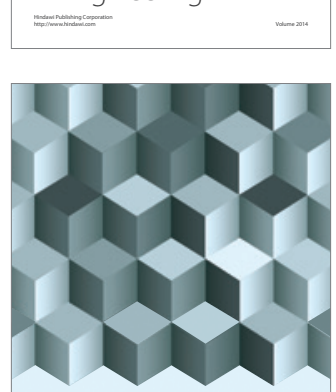

Journal of

Function Spaces
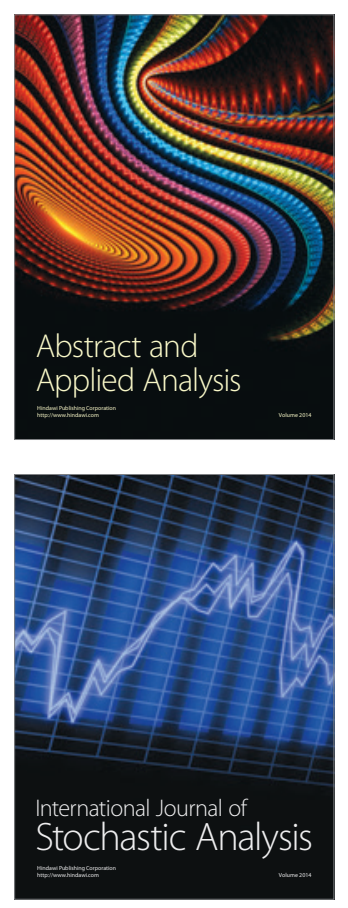

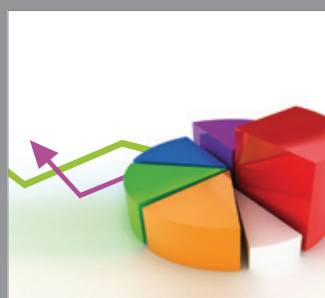

ournal of

Probability and Statistics

Promensencen
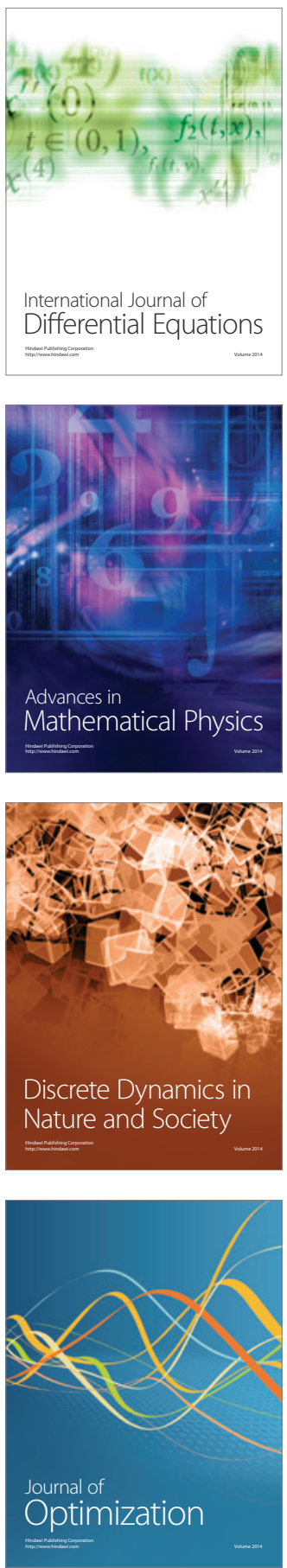